\title{
Pesca del bacalao de profundidad (Dissostichus eleginoides), efectuada por la flota chilena en torno a la isla Georgia del Sur (1991/1992)*
}

\author{
PATRICIO ARANA E., MARCELO ARREDONDO A. y VITTORIO VENTURINI M. \\ Escuela de Ciencias del Mar \\ Universidad Católica de Valparaíso \\ Casilla 1020, Valparaíso, Chile
}

RESUMEN. En este estudio se analizan faenas de pesca de la flota espinelera chilena, orientada a la extracción del bacalao de profundidad (Dissostichus eleginoides) en la subárea administrativa 48.3 de la Convención para la Conservación de los Recursos Vivos Marinos Antárticos (CCRVMA) (Isla Georgia del Sur). La flota capturó alrededor del 80\% de la cuota total establecida en esa subárea, desde fines de diciembre de 1991 hasta el cierre de la pesquería, el 10 de marzo de 1992.

A través del posicionamiento de los lances, se distinguen dos caladeros claramente diferenciables: uno al norte de la isla Georgia del Sur, que incluye los islotes Black y Shag Rocks, y otro al sur de esa isla, concentrándose los lances entre los 1.100 y los $1.450 \mathrm{~m}$ de profundidad. El esfuerzo total aplicado por la flota fue de 2,71 millones de anzuelos, la captura de 2.883 ton y la CPUE promedio alcanzó a $1,06 \mathrm{~kg} /$ anzuelo.

En ese período se midió la longitud estándar a 8.340 ejemplares, efectuados a intervalos de $5 \mathrm{~cm}$, los que fueron convertidos a longitud total. El rango de tallas en las capturas fue de 40-145 cm en machos y de 40-200 cm en hembras. Los valores promedios fueron $96,7 \mathrm{~cm}$ y $108,8 \mathrm{~cm}$, respectivamente en machos y hembras.

La evaluación del stock se efectuó mediante dos aproximaciones metodológicas: análisis de seudocohorte y aplicación del método de DeLury. Se definieron Capturas Totales Permisibles (CTP), utilizando diferentes combinaciones de $\mathrm{F}_{0,1}$, longitud de primera captura y mortalidad natural. Con el método de DeLury, se obtuvieron capturas totales permisibles entre 1.085 y 5.768 ton, mientras que a través del análisis de cohorte, la CTP fluctúa entre 7.832 y 8.210 ton.

Palabras claves: Evaluación de poblaciones, bacalao de profundidad, Dissostichus eleginoides, actividades pesqueras, región antártica, Isla Georgia del Sur.

\section{Fishing of the Patagonian toothfish (Dissostichus eleginoides) by the Chilean fleet around South Georgia Island (1991/1992)}

\begin{abstract}
This study analyses fisheries activities carried out for the first time by the Chilean longline fishery targeting the Patagonian toothfish (Dissostichus eleginoides) in the Convention for the Conservation of the Antarctic Marine Living Resources (CCAMLR) Statistical Subarea 48.3 (South Georgia Island). From the end of December 1991 until the closing of the fishery on the 10th March 1992, the fleet caught around 80\% of the TAC set for this subarea.

From the location of the hauls, two separate grounds could be clearly distinguished: the first one at the north of South Georgia Island, including Black and Shag rocks, and the second one at the south ofthe island, hauls being made at depths
\end{abstract}

* Documento presentado en la reunión del Grupo de Trabajo de Evaluación de Poblaciones de Peces de la Convención para la Conservación de los Recursos Vivos Marinos Antárticos (CCRVMA), Horbart, Tasmania, Australia, 13-22 de octubre de 1992. 
between 1100 and $1450 \mathrm{~m}$. The effort applied by the Chilean fleet totalled 2.71 million hooks, 2883 tones were caught at an average CPUE of $1.06 \mathrm{~kg} / \mathrm{hook}$.

During this period, the standard length of 8340 specimens was measured at $5 \mathrm{~cm}$ intervals. The catch lengths distributions ranged between 40 and $145 \mathrm{~cm}$ for males and 40 and $200 \mathrm{~cm}$ for females. The average length was estimated al $96.7 \mathrm{~cm}$ for males and $108.8 \mathrm{~cm}$ for females.

The stock assessment was made by using two methodological approaches: pseudo-cohort analysis and DeLury's method. According to the biomass obtained by each method, TAC were established, using different combinations of $\mathrm{F}_{0,1}$, total length at first catch and natural mortality. According to DeLury's method, TACs ranged between 1085 and 5768 tones, whereas the cohort analysis gave TACs fluctuating between 7832 and 8210 tones.

Key words: Stock assessment, Patagonian toothfish, Dissostichus eleginoides, fishing activities, Antarctic zone, South Georgia Island.

\section{INTRODUCCION}

El bacalao de profundidad (Dissostichus eleginoides) es un recurso íctico abisobentónico de amplia distribución circumpolar antártica, que ha suscitado interés para su extracción comercial en todos los lugares en que se ha establecido la presencia de esta especie. En Chile las actividades orientadas a la extracción de este recurso han permitido el desarrollo de una importante pesquería desde comienzos de la década del 70. Por dicha razón, al saberse que embarcaciones espineleras chilenas podrían realizar faenas extractivas sobre el bacalao de profundidad en la subárea administrativa 40.3 (Isla Georgia del Sur), en el ámbito de competencia de la Convención para la Conservación de los Recursos Vivos Marinos Antárticos (CCRVMA), numerosas empresas enviaron prontamente embarcaciones a esa zona de pesca.

El éxito y experiencia logrados en esta primera incursión masiva a la zona antártica hacen necesario que Chile no sólo mantenga su liderazgo en el volumen capturado, sino que también tenga una presencia activa en la investigación biológico-pesquera de este recurso y en la determinación de las cuotas anuales de captura. Así también, la expansión de las ope raciones y la obtención de mayores capturas de $D$. eleginoides, permiten consolidar la posición del país entre los abastecedores mundiales de este producto.

El principal objetivo del presente trabajo es el reunir y procesar los registros de captura y esfuerzo, así como de los muestreos biológicos efectuados a bordo de los buques chilenos, con el fin de obtener una primera visión de las acciones desarrolladas por esta flota en la región antártica. Adicionalmente, se pretende analizar la información globalizada de las capturas totales realizadas durante la temporada 1991/ 1992 en esa subárea, con el fin de aproximarse a la situación del recurso y su potencialidad.

\section{MATERIALES Y METODOS}

\section{Aspectos generales}

En este estudio se analiza la información biológicopesquera recopilada sobre las actividades extractivas del bacalao de profundidad (Dissostichus eleginoides), efectuadas en la subárea administrativa 48.3 (isla Georgia del Sur), en el ámbito de competencia de la Convención para la Conservación de los Recursos Vivos Marinos Antárticos (CCRVMA). Dichas operaciones se realizaron entre el 02 de noviembre de 1991 y el 10 de marzo de 1992, cuando se declaró el cierre de esa pesquería, al ser completada la cuota de 3.500 ton, asignada para la temporada.

La información procesada en el presente documento corresponde a los registros de esfuerzo, captura y captura por unidad de esfuerzo (CPUE) de la flota de embarcaciones espineleras chilenas que operó en esa subárea. Así también, se utilizaron los informes estadísticos de la Secretaría Ejecutiva de la CCRVMA (Hobart, Tasmania), con el fin de proyectar los valores a toda la flota que efectuó faenas extractivas en esa subárea y temporada de pesca. Los datos entregados por la CCRVMA se refirieron a las capturas comunicadas por cada país, agrupadas en períodos de 5 días.

En forma complementaria, se analizan los registros de distribuciones de frecuencias de tallas que se obtuvieron en cada una de las embarcaciones que trabajaron en esa pesquería. Con este fin se realizaron muestreos de las capturas, midiéndose la longitud a un minimo de 30 ejemplares por lance, en intervalos de $5 \mathrm{~cm}$.

Cabe destacar que en los muestreos realizados para establecer la talla de los ejemplares capturados, su efectuaron midiendo la longitud estándar, por lo que fue necesario transformar los registros a tallas 
totales a través de un factor de proporcionalidad morfológica. Por otra parte, en atención a que las mediciones se realizaron sin diferenciar el sexo de los individuos, se estableció el número de machos y hembras presentes en cada rango de longitud, a través de un procedimiento de separación que considera la proporción sexual a la talla, relación que responde a un patrón específico del recurso. Esta característica ha sido demostrada en otras especies y se ha sugerido su utilización con este mismo fin (Gaete y Arana, 1985; García y Le Reste, 1983; Arredondo, 1991). Para obtener este patrón, se utilizaron las distribuciones de frecuencias de longitudes establecidas separadamente para cada sexo en las faenas de pesca exploratoria realizadas por el B/ $\mathrm{P}$ «Friosur $\mathrm{V} »$ en esta misma área de estudio, entre febrero y marzo de 1991.

Adicionalmente, se efectuó un análisis de las distribuciones de frecuencia de tallas proporcionadas por Gasyukov et al. (1991) quien entrega series de longitud correspondientes a las capturas registradas desde la temporada 1984/85 hasta la 1990/91. En forma conjunta, se incluyó la información de la temporada recién pasada (1991/92), referidas en Arana et al. (1992). En ambos casos, se efectuó una disgregación uniforme mediante una interpolación lineal entre dos marcas de clase consecutivas, obteniendo la frecuencia correspondiente en rangos de $1 \mathrm{~cm}$.

Por otra parte, con el objeto de determinar el peso promedio de los ejemplares capturados, así como la contribución de cada sexo en la captura total, se calculó el aporte en peso correspondiente a machos y hembras, de acuerdo a la respectiva distribución de frecuencias de tallas registradas en las capturas. Para realizar estos cálculos se utilizaron las funciones talla-peso establecidas por Aguayo y Cid (1991). Finalmente, el peso promedio individual en cada sexo, se obtuvo dividiendo el aporte en peso total por el número de ejemplares considerados.

\section{Análisis del esfuerzo, captura y CPUE}

En virtud de que la información proporcionada por el Servicio Nacional de Pesca (SERNAP) incluyó una amplia variedad de datos y que las capturas registradas por las embarcaciones chilenas alcanzaron a alrededor del $80 \%$ de la cuota total asignada, fue a partir de esta fuente que se efectuaron los análisis de esfuerzo, captura y rendimiento. De este modo, se caracterizó la flota de acuerdo al tamaño de la embarcación, al arte empleado y al sistema de preservación utilizado. Asimismo, se ubicó geográficamente la posición de los lances para establecer la ubicación de caladeros de pesca y así procesar estos datos en forma global y separadamente para cada uno de ellos.

Indistintamente, para realizar el análisis de la información, los datos fueron agrupados en base diaria. Luego, debido a la gran dispersión observada y para obtener una representación suavizada de las progresiones obtenidas, se procedió a aplicar promedios móviles en agrupamientos de tres datos.

\section{Crecimiento}

La función de crecimiento utilizada en el presente documento corresponde a la calculada por Arana et al. (1992) a partir de las claves talla vs. edad de este recurso, establecidas por Aguayo y Cid (1991) para cada sexo. Es así que las ecuaciones matemáticas estimadas para machos y hembras son las siguientes:

$$
\begin{array}{ll}
\text { Machos } & \mathrm{L}_{\mathrm{t}}=150,82\left[1-\mathrm{e}^{-0,1052(\mathrm{t}+0,0416)}\right] \\
\text { Hembras } & \mathrm{L}_{\mathrm{t}}=178,37\left[1-\mathrm{e}^{-0,0782(\mathrm{t}+0,4018)}\right] \\
\text { Ambos sexos } & \mathrm{L}_{\mathrm{t}}=164,78\left[1-\mathrm{e}^{-0,0965(\mathrm{t}+0,4310)}\right]
\end{array}
$$

En cuanto al crecimiento en peso, a partir de la relación longitud peso proporcionada por Aguayo y Cid (1991), Arana et al. (1992) establecieron las siguientes ecuaciones:

$$
\begin{aligned}
& \text { Machos } \quad \mathrm{W}_{\mathrm{t}}=27,820\left[1-\mathrm{e}^{-0,1052(\mathrm{t}+0,0416)}\right]^{2,486555} \\
& \text { Hembras } \quad \mathrm{W}_{\mathrm{t}}=41,876\left[1-\mathrm{e}^{-0,0782(\mathrm{t}+0,4018)}\right]^{2,406555} \\
& \text { Ambos sexos } \mathrm{W}_{\mathrm{t}}=35,584\left[1-\mathrm{e}^{-0,0965(\mathrm{t}+0,4310)}\right]^{2,558653}
\end{aligned}
$$

Con los resultados señalados, los citados autores determinaron una expectativa de vida de 28 y 38 años, respectivamente en los machos y en las hembras de esta especie.

Ahora bien, en atención a que las claves talla $v s$. edad establecida por Aguayo y Cid (1991) se obtuvieron a partir de la lectura de escamas, es necesario destacar los resultados de análisis lepidométrico efectuados por Hureau y Ozouf-Costaz (1980/81). Estos autores señalan que debido a que el desove de esta especie ocurre en mayo, «el primer invierno de vida del pez no involucra la formación de una marca anual reconocible en las escamas». Por esta razón, el primer 
anillo observado (clase I), corresponde al segundo invierno después del desove; lo que involucra que al momento de la captura, los ejemplares posean alrededor de 10 a 11 meses más de edad que la indicada en sus escamas. Lo anteriormente planteado, afecta la función de crecimiento, pero sólo en su parámetro $\mathrm{t}_{\mathrm{o}}$, el que se verá incrementado en una unidad. Esto redunda en valores de $\mathrm{t}_{\mathrm{o}}$ recalculados de 0,9584; 0,5982 y 1,4310 , respectivamente en machos, hembras y en ambos sexos en conjunto.

\section{Mortalidades}

Para los análisis efectuados en este estudio se utilizaron las estimaciones de las tasas instantáneas de mortalidad natural (M) y total (Z) efectuadas por Arana et al. (1992). Con este fin se emplearon métodos bioanalógicos (Pauly, 1980; Rikhtery Efanov, 1976 y Roff, 1984), analíticos (Bevertony Holt, 1956; la modificación propuesta por Pauly, 1983 y Ssentongo y Larkin, 1973) y basados en un segmento de la curva de captura (Van Sickle, 1977; Pauly, 1984; Jones y Van Zalinge, 1982). El cálculo de la tasa instantánea de mortalidad por pesca se efectuó como la diferencia entre Z y M. Los parámetros utilizados para aplicar estas metodologias y los valores obtenidos se presentan en la Tabla 1.

\section{Evaluación del recurso}

La evaluación de la población del bacalao de profundidad que habita en las aguas adyacentes a la isla Georgia del Sur (subárea 48.3), se realizó mediante análisis de cohorte (Pope, 1972) y a través del modelo simple de DeLury (Chapman, 1972; Ricker, 1975). Cabe destacar, que al no disponer de información que permitiera establecer una matriz histórica de captura por grupos de edades, el primer método se empleó utilizando únicamente las capturas estructuradas por edad correspondiente a la temporada 1991/1992. Ello se debe a que la flota chilena inició actividades en la región en esa temporada, no contándose con información previa. En relación a la aproximación metodológica antes indicada, es preciso señalar que este tipo de análisis se efectúa bajo el supuesto de que la población permanece en equilibrio.

La información básica utilizada para efectuar dicha evaluación corresponde a las frecuencias de longitudes obtenidas en los muestreos diarios de la pesca comercial realizada por las embarcaciones chilenas, sirviendo éstas como base para estructurar la captura en edades obtenida por toda la flota que operó en esa temporada. Las capturas por grupo de edad, se obtuvieron mediante el empleo de las claves «Tallaedad» obtenidas por Aguayo y Cid (1991) y, además, reescalando las mismas en un año, de acuerdo a la corrección que habría que efectuar en las lecturas lepidométricas realizadas en el bacalao de profundidad (Hureau y Ozouf-Costaz, 1980/81).

Por otra parte, para aplicar el método de DeLury se utilizaron las capturas por unidad de esfuerzo (CPUE), en número de ejemplares por millón de anzuelos calados, obtenidas en períodos de cinco días por los buques espineleros chilenos que operaron en la región durante la temporada 1991/1992. Los valores de rendimientos por anzuelo fueron regresionados con las capturas acumuladas totales obtenidas por todos los buques que participaron en esta pesquería, corregidas por la tasa instantánea de mortalidad natural, que afecta al recurso en el período correspondiente. En atención a que la flota chilena capturó alrededor del $80 \%$ de la cuota de pesca establecida en la temporada, el análisis se efectuó bajo el supuesto de que los restantes buques, de los cuales se registraron capturas pero no se obtuvo información de esfuerzo, presentan igual CPUE que la alcanzada por la flota nacional.

\section{Captura Total Permisible (CTP)}

Las estimaciones de CTP de bacalao de profundidad proyectada para la temporada 1992/1993, se efectuaron a partir del análisis de cohorte y, además, utilizando la biomasa obtenida por el método de DeLury. En el primero de ellos, se empleó el vector de pesos medios a la edad, obtenido con la relación edad-peso establecida a partir de los parámetros de crecimiento y la relación talla-peso de Aguayo y Cid (1991).

Debido a la incertidumbre observada en los parámetros biológico-pesqueros disponibles sobre esta especie, se efectuaron estimaciones de CTP para machos y hembras en forma separada, de acuerdo a distintos valores de $\mathrm{F}_{0,1}$ estimados a través del método de Thompson y Bell (1934). De igual manera, al trabajar sobre la población obtenida con el método de DeLury, para el cálculo de la captura total permisible se procedió a utilizar varios valores de mortalidad por pesca óptima, calculados estos a partir de los modelos de rendimiento por recluta de Thompson y Bell, así como por el de Beverton y Holt.

Las tasas instantáneas de mortalidad por pesca óptima se calcularon conjugando diferentes mortalidades naturales $(0,06 ; 0,10 ; 0,11 ; 0,12$ y 0,18$)$ y eda- 
Tabla 1. Estimados más probables de mortalidad natural (M) y de mortalidad total (Z) (Arana et al., 1992)

\begin{tabular}{|c|c|c|c|c|c|c|c|}
\hline \multirow{2}{*}{\multicolumn{2}{|c|}{ MORTALIDAD / METODO - }} & \multicolumn{2}{|c|}{ PARAMETRO L } & UTILIZADO & \multicolumn{3}{|c|}{ MORTALIDAD (SEXO) } \\
\hline & & \multicolumn{2}{|c|}{ ESCRIPCION MACHOS } & HEMBRAS & MACHOS & HEMBRAS & AMBOS \\
\hline \multicolumn{8}{|c|}{ MORTALIDAD NATURAL (M) } \\
\hline \multicolumn{8}{|c|}{ METODOS BIOANALOGICOS } \\
\hline \multicolumn{2}{|c|}{ - PAULY (1980) } & temp & 4,0 & 4,0 & 0,11 & 0,09 & 0,10 \\
\hline \multicolumn{2}{|c|}{ - RIKHTER y EFANOV (1976) } & TMS & $9,5 / 10,5$ & $9,5 / 10,5$ & $0,13-0,15$ & $0,11-0,14$ & $0,12-0,15$ \\
\hline \multicolumn{2}{|c|}{ - ROFF (1984) (M máx) } & LMS & 100 & 100 & $0,16-0,19$ & $0,17-0,20$ & $0,16-0,20$ \\
\hline \multicolumn{8}{|c|}{ MORTALIDAD TOTAL $(Z)$} \\
\hline \multicolumn{8}{|c|}{ METODOS ANALITICOS } \\
\hline \multicolumn{2}{|c|}{ - BEVERTON y HOLT (1956) } & $\operatorname{Lc}(50 \%)$ & 80 & 90 & 0,49 & 0,39 & 0,48 \\
\hline \multicolumn{2}{|c|}{ - Modificación de PAULY (1983) } & $\operatorname{tc}(50 \%)$ & $7,5 / 8,5$ & $8,5 / 9,5$ & 0,49 & 0,39 & 0,48 \\
\hline \multicolumn{2}{|c|}{ - SSENTONGO y LARKIN (1973) } & $\operatorname{Lc}(50 \%)$ & 80 & 90 & 0,54 & 0,43 & 0,53 \\
\hline & & LMED & 96,66 & 108,84 & & & \\
\hline \multicolumn{8}{|c|}{$\begin{array}{l}\text { METODOS BASADOS EN UN SEGMENTO } \\
\text { DE LA CURVA DE CAPTURA }\end{array}$} \\
\hline \multicolumn{2}{|c|}{ - Van SICKLE (1977) } & INC & 3,669 & 3,669 & $0,47-0,48$ & 0,32 & 0,38 \\
\hline \multicolumn{2}{|c|}{ - PAULY (1983) } & - & - & - & $0,37-0,38$ & 0,29 & 0,31 \\
\hline \multicolumn{2}{|c|}{ - JONES y VAN ZALINGE (1982) } & - & - & - & 0,88 & $0,48-0,49$ & 0,53 \\
\hline & Machos & $\mathrm{L}_{\infty}=150,82$ & \multicolumn{2}{|c|}{$\mathrm{k}=0,1052$} & \multicolumn{3}{|c|}{$t_{0}=-0,0416 / 0,9584$} \\
\hline & Hembras & \multicolumn{2}{|c|}{$\mathrm{L}_{\infty}=178,37$} & 0,0782 & \multicolumn{2}{|c|}{$t_{0}=-0,4018 / 0,5900$} & \\
\hline temp & \multicolumn{3}{|c|}{$\begin{array}{l}: \text { temperatura ambiente en el rango de distribu- } \\
\text { ción del recurso }\left({ }^{*} \mathrm{C}\right)\end{array}$} & $\begin{array}{ll}\mathrm{INC} & : \\
\operatorname{Lc}(50 \%) & :\end{array}$ & \multicolumn{3}{|c|}{$\begin{array}{l}\text { : incremento medio anual de longitud }(\mathrm{cm}) \\
: \text { longitud de primera captura }(\mathrm{cm})\end{array}$} \\
\hline TMS & \multicolumn{3}{|c|}{$:$ edad de primera madurez sexual (años) } & $\operatorname{tc}(50 \%):$ & \multicolumn{3}{|c|}{ edad de primera captura (años) } \\
\hline LMS & \multicolumn{3}{|c|}{ : longitud de primera madurez sexual $(\mathrm{cm})$} & LMED : & longitud media & de las captura & $(\mathrm{cm})$ \\
\hline
\end{tabular}

des de primera captura (5-10 años o 6-11 años). En el caso de la estimación de $\mathrm{F}_{0,1}$ a través del modelo de rendimiento por recluta de Beverton y Holt, se utilizaron los parámetros de crecimiento estimados en este trabajo en forma conjunta para ambos sexos.

\section{Tratamiento de la información}

El procesamiento de la información se efectuó mediante los Programas DISFRE (Distribución de frecuencias de tallas), RAMOR (Resultados y análisis de mortalidades), LFSA (Length-based fish stock assessment; P. Sparre); Virtual Population Analysis (Versión 1) y TAC Based Projection (Versión 2) de la
CCAMLR, y diversos programas desarrollados especialmente con este objetivo. En los procesos se utilizaron computadores Acer PC-AT 910.

\section{RESULTADOS}

\section{Captura, esfuerzo y CPUE}

\section{Capturas}

Los países participantes, Bulgaria, Chile y la URSS, capturaron el total del tonelaje asignado entre fines de noviembre de 1991 y el 10 de marzo de 1992, fecha en que se declaró cerrada la pesquería (Fig. 1). 
La fracción extraída por cada una de estas flotas, de acuerdo a cifras de la CCRVMA, correspondió al $4 \%, 79 \%$ y $17 \%$, respectivamente. No obstante lo anterior, en base a estadísticas de lances individuales, proporcionadas por el SERNAP, la participación de la flota chilena en las capturas globales informadas alcanzó al $82 \%$ de la cuota asignada.

Las embarcaciones chilenas que efectuaron lances en la subárea 48.3 durante la temporada 1991/ 1992, correspondieron a naves de 250 a 1.000 TRB. $\mathrm{El}$ arte empleado por la totalidad de ellos fue el espinel de fondo de tendido horizontal y la preservación de la captura se efectuó a través del congelado.

Las faenas extractivas se concentraron en zonas aledañas a la isla Georgia del Sur, distinguiéndose en la región dos caladeros de pesca, uno al sur de dicho territorio insular y otro al norte, que incluye las aguas circundantes a las Black y Shag Rocks (Fig. 2). El número de lances efectuados en la subárea fue de 309 , correspondiendo 226 al caladero norte y 83 al sur (Fig. 3). La profundidad media de calado fluctuó desde los $970 \mathrm{~m}$ hasta los $2.000 \mathrm{~m}$, concentrándose los lances entre los $1.100 \mathrm{~m}$ y $1.450 \mathrm{~m}$. Las faenas de pesca tuvieron un régimen de operación diario consistente en el calado del espinel por la noche y virado al amanecer.
Las faenas extractivas de la flota chilena, de acuerdo a la información de lances individuales, generaron capturas totales en la subárea de 2.883 ton, registrándose el 67,7\% (1.94 9 ton) en el caladero norte y el 32,4\% (934 ton) en el sur (Tabla 2). Los registros diarios de pesca en el caladero norte fueron de alrededor de 30 ton/día hasta mediados de febrero, experimentando un alza hacia fines de la temporada a 50 ton/día. En el caladero sur, en cambio, las capturas registraron un fuerte incremento a 50 ton/día en el inicio de las operaciones, para posteriormente decrecer y mantenerse estables en 15 ton/día. Asimismo, las capturas de ambos caladeros en forma conjunta, sufren fluctuaciones de 20 a 50 ton/día hasta la primera quincena de febrero, aumentando con posterioridad de 40 a 60 ton/día (Figs. 4,7 y 10).

\section{Esfuerzo}

El esfuerzo total aplicado por la flota chilena fue de 2.709.650 anzuelos, habiéndose calado 817.660 $(30,2 \%)$ en el caladero sur y $1.891 .990(69,8 \%)$ en el norte (Tabla 2). El número de anzuelos calados diariamente en ambos caladeros se fue incrementando en el transcurso del tiempo desde 15.000 unidades al inicio de la temporada, hasta alrededor de 60.000 anzuelos hacia el fin de ella (Figs. 5,8 y 11).

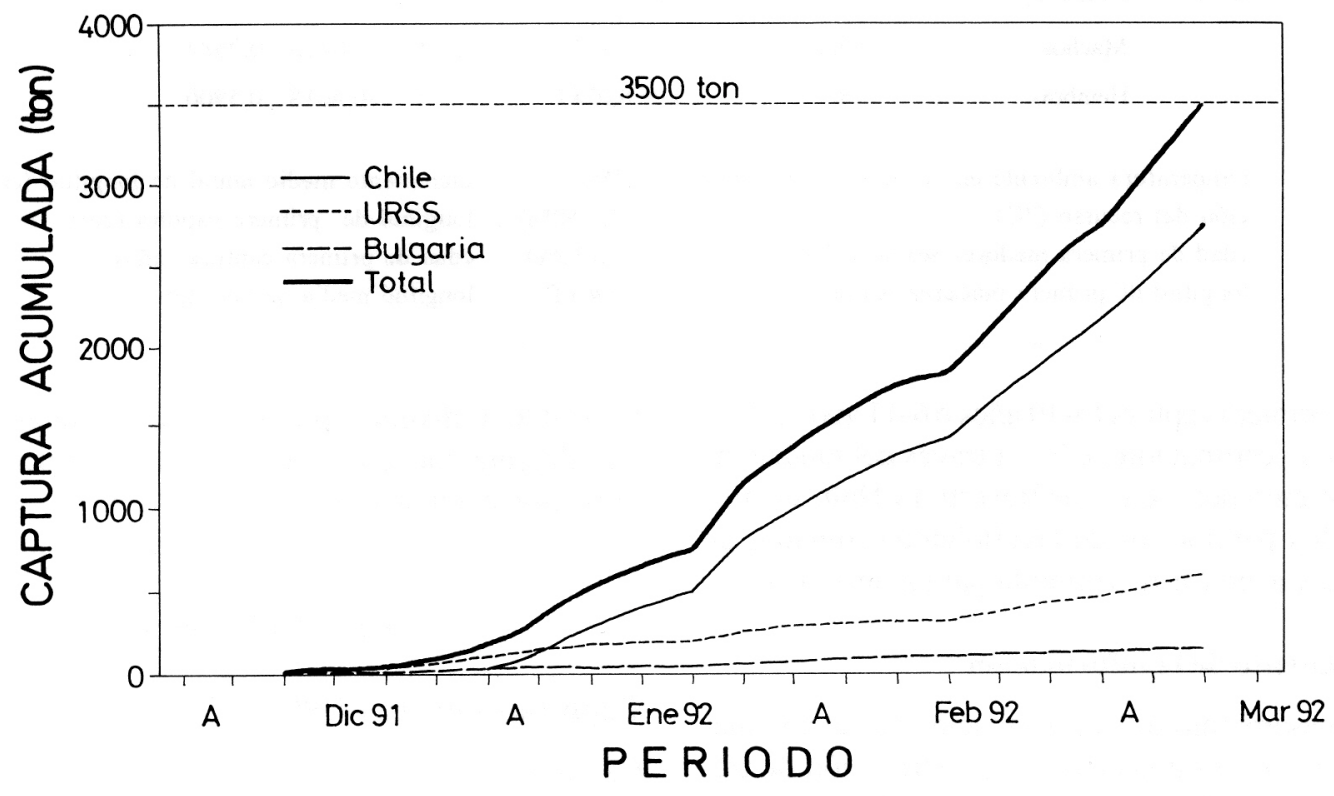

Figura 1. Capturas acumuladas de bacalao de profundidad, registradas en la subárea 48.3 durante la temporada 1991/1992, por las flotas de los diferentes países participantes en la pesquería, proyectando la cifra del último período de acuerdo a la cuota fijada de 3.500 ton. Fuente: Datos estadísticos de la Secretaría Ejecutiva de la CCRVMA. 


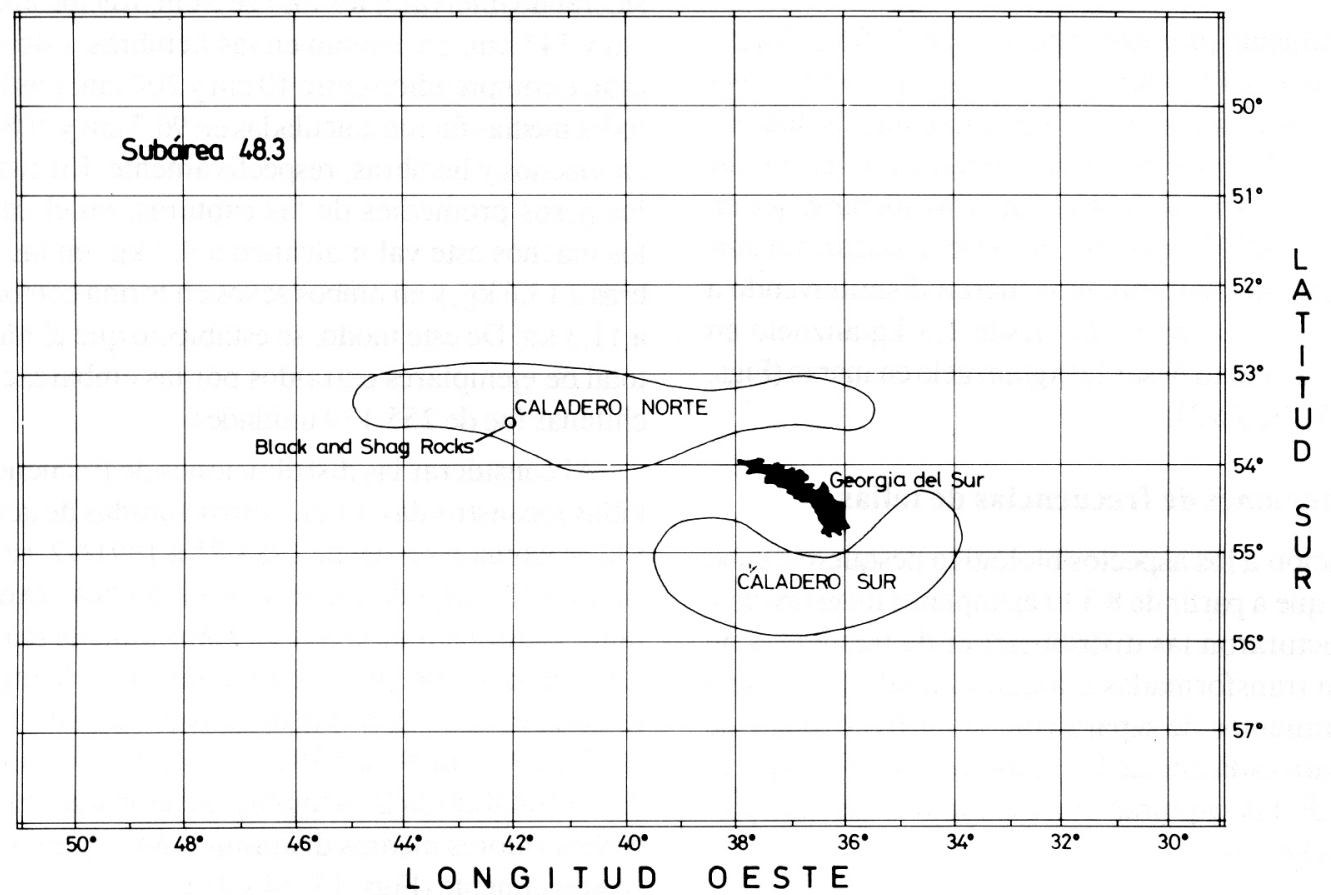

Figura 2. Ubicación de los caladeros de pesca utilizados por la flota chilena durante la temporada 1991/1992 en la subárea 48.3.

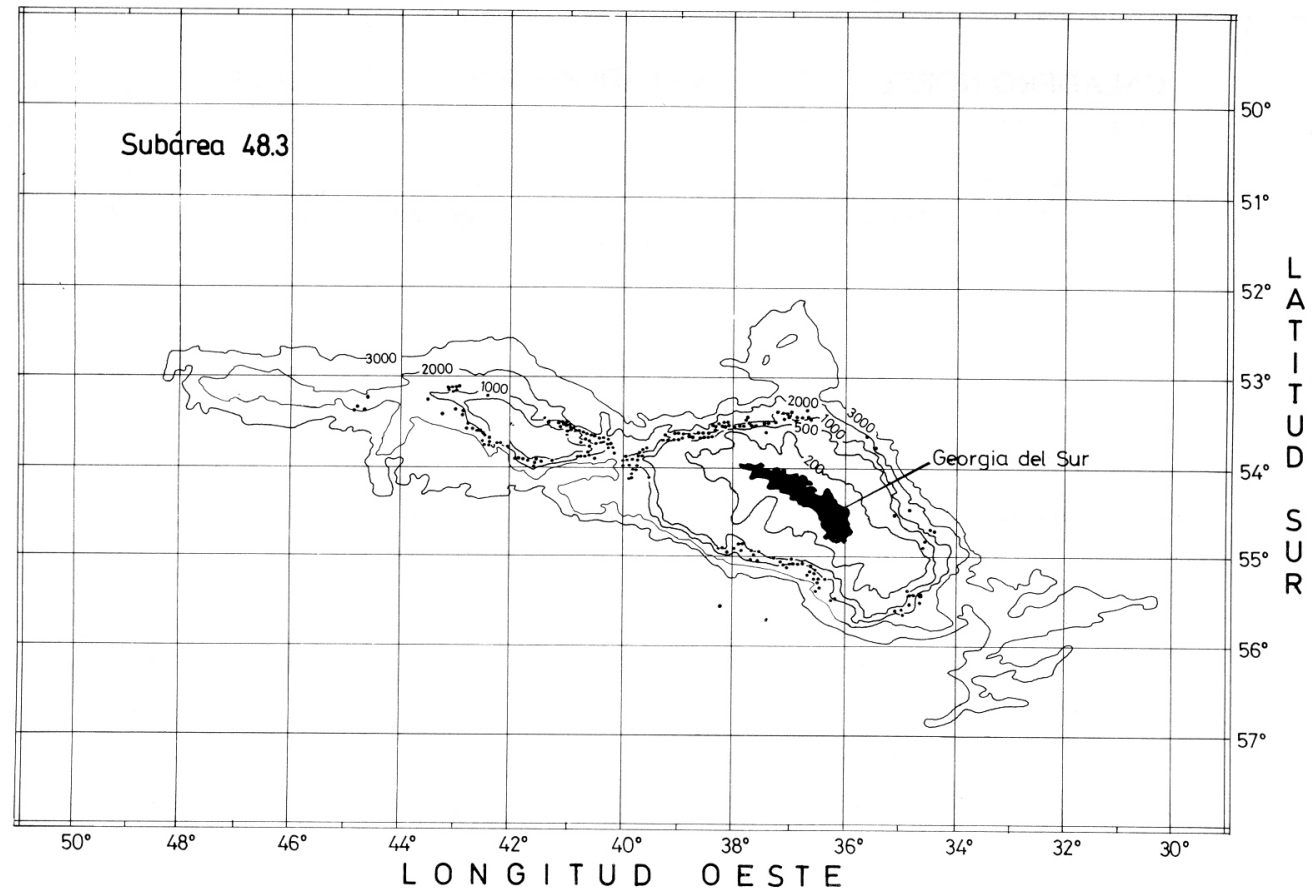

Figura 3. Lances efectuados por la flota chilena en la subárea 48.3 durante la temporada 1991/1992, destinados a la captura del bacalao de profundidad. 


\section{Rendimiento}

El rendimiento promedio obtenido por la flota chilena en la subárea 48.3 durante la temporada 1991/1992 fue de $1,06 \mathrm{~kg} /$ anzuelo, registrándose ligeras diferencias entre los dos caladeros, puesto que en el sur alcanzó una cifra de $1,14 \mathrm{~kg} /$ anzuelo mientras que en el norte a 1,03 kg/anzuelo. Se debe destacar, sin embargo, que los rendimientos fueron disminuyendo a lo largo de la temporada, desde $1,3 \mathrm{~kg} /$ anzuelo en diciembre y enero hasta $1,0 \mathrm{~kg} /$ anzuelo en marzo (Figs. 6,9 y 12) (Tabla 2).

\section{Distribuciones de frecuencias de tallas}

En relación a los aspectos biológico pesqueros, cabe señalar que a partir de 8.340 ejemplares muestreados, se estructuraron las distribuciones de frecuencia de talla, ya transformadas a longitud total, y en base a procedimientos de separación de las frecuencias de tallas para cada uno de los sexos, se determinó que el $59,0 \%$ de las capturas correspondió a machos y el $41,0 \%$ a hembras.
El rango de tallas observado en los machos, considerando intervalos de talla de $5 \mathrm{~cm}$, fluctuó entre $40 \mathrm{~cm}$ y $145 \mathrm{~cm}$, en cambio en las hembras, este rango estuvo comprendido entre $40 \mathrm{~cm}$ y $200 \mathrm{~cm}$. Las longitudes medias fueron calculadas en $96,7 \mathrm{~cm}$ y $108,8 \mathrm{~cm}$ en machos y hembras, respectivamente. En cuanto a los pesos promedios de las capturas, en el caso de los machos este valor alcanzó a 9,7 kg, en las hembras a $13,6 \mathrm{~kg}$, y en ambos sexos en forma combinada a $11,3 \mathrm{~kg}$. De este modo, se estableció que el número total de ejemplares extraídos por las embarcaciones chilenas fue de 255.139 unidades.

Al considerar las distribuciones de frecuencia de tallas reconstruidas a $1 \mathrm{~cm}$, estructuradas de acuerdo a la proporción sexual de 1984/85 a 1991/92, se aprecia que el rango de longitudes en ambos sexos durante la última temporada es notoriamente más amplio al observado en los años anteriores. Asimismo, se observa que las tallas medias aumentaron entre 1985 y 1991 ; de 85,2 a $102,5 \mathrm{~cm}$ en los machos y de 97,9 a $108,8 \mathrm{~cm}$ en las hembras. Sin embargo, en 1992, dichos valores medios disminuyeron, según se indicó previamente (Figs. 13, 14 y 15).

Tabla 2. Resumen de estadísticas de captura, esfuerzo y rendimiento obtenidos por la flora chilena en la subárea 48.3, durante la temporada 1991/1992

\begin{tabular}{|c|c|c|c|c|c|c|c|c|c|}
\hline \multirow{2}{*}{ MES } & \multicolumn{3}{|c|}{ CALADERO NORTE } & \multicolumn{3}{|c|}{ CALADERO SUR } & \multicolumn{3}{|c|}{ AMBOS CALADEROS } \\
\hline & $\begin{array}{c}\text { Captura } \\
\text { (ton) }\end{array}$ & $\begin{array}{l}\text { Esfuerzo } \\
\left(\mathrm{N}^{\circ} \mathrm{de}\right. \\
\text { anzuelos) }\end{array}$ & $\begin{array}{c}\text { CPUE } \\
\text { (kg/anz) }\end{array}$ & $\begin{array}{c}\text { Captura } \\
\text { (ton) }\end{array}$ & $\begin{array}{l}\text { Esfuerzo } \\
\left(\mathrm{N}^{\circ} \text { de }\right. \\
\text { anzuelos) }\end{array}$ & $\begin{array}{c}\text { CPUE } \\
\text { (kg/anz) }\end{array}$ & $\begin{array}{c}\text { Captura } \\
\text { (ton) }\end{array}$ & $\begin{array}{l}\text { Esfuerzo } \\
\text { ( } \mathrm{N}^{\circ} \text { de } \\
\text { anzuelos) }\end{array}$ & $\begin{array}{c}\text { CPUE } \\
\text { (kg/anz) }\end{array}$ \\
\hline $\begin{array}{c}\text { Dic } \\
(1991)\end{array}$ & - & - & - & 34 & 26.980 & 1,26 & 34 & 26.980 & 1,26 \\
\hline $\begin{array}{c}\text { Ene } \\
(1992)\end{array}$ & 493 & 393.440 & 1,25 & 566 & 416.280 & 1,36 & 1.059 & 809.720 & 1,31 \\
\hline $\begin{array}{c}\text { Feb } \\
(1992)\end{array}$ & 967 & 1.026 .550 & 0,94 & 210 & 257.400 & 0,82 & 1.178 & 1.283 .950 & 0,92 \\
\hline $\begin{array}{c}\text { Mar } \\
(1992)\end{array}$ & 489 & 472.000 & 1,04 & 123 & 117.000 & 1,05 & 612 & 589.000 & 1,04 \\
\hline $\begin{array}{c}\text { TOTAL } \\
(1991 / 92)\end{array}$ & 1.948 & 1.891 .990 & 1,03 & 933 & 817.660 & 1,14 & 2.883 & 2.709 .650 & 1,06 \\
\hline
\end{tabular}




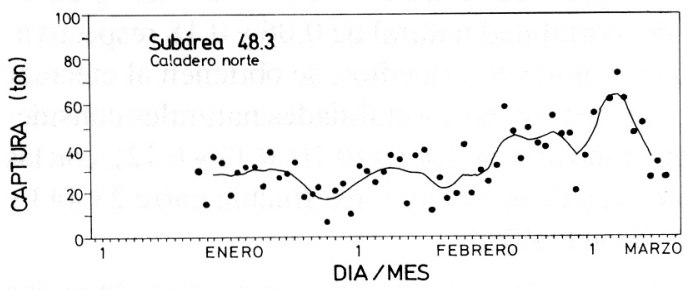

Figura 4. Captura de bacalao de profundidad registrada por la flota chilena en la subárea 48.3, durante la temporada 91/92.

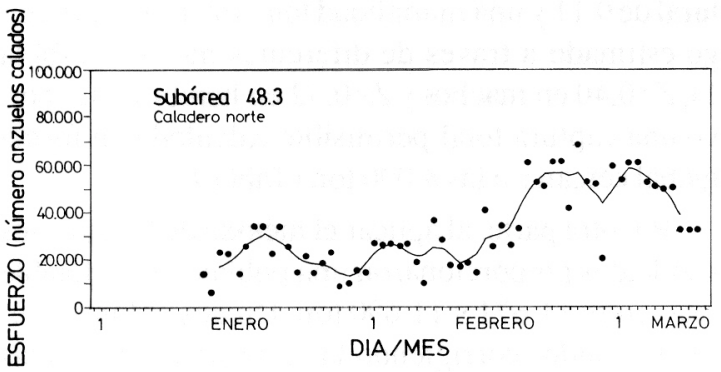

Figura 5. Esfuerzo aplicado por la flota chilena para la captura de bacalao de profundidad en la subárea 48.3, durante la temporada 91/92.

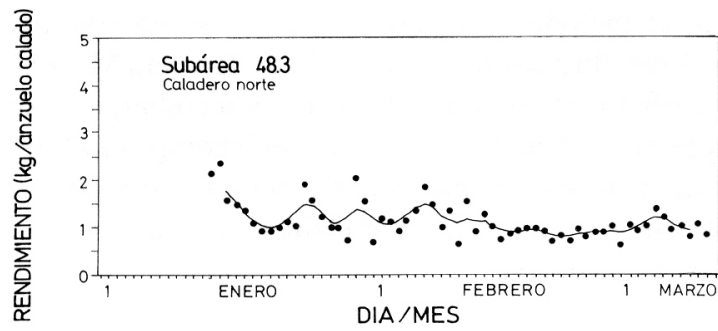

Figura 6. Rendimiento obtenido por la flota chilena en la captura de bacalao de profundidad en la subárea 48.3, durante la temporada 91/92.

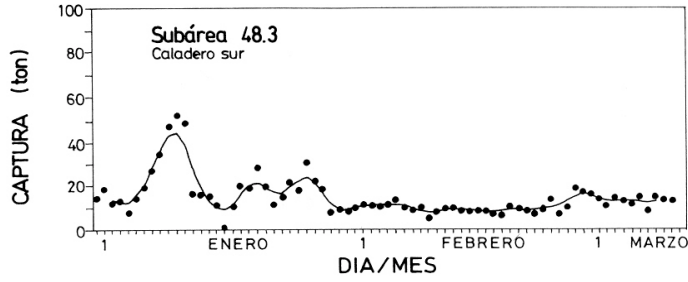

Figura 7. Captura de bacalao de profundidad registrada por la flota chilena en la subárea 48.3, durante la temporada 91/92.

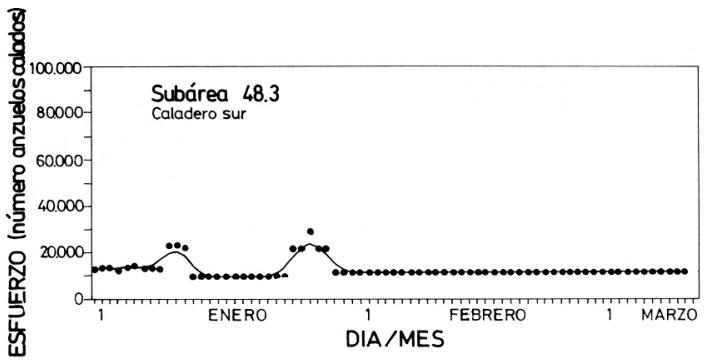

Figura 8. Esfuerzo aplicado por la flota chilena para la captura de bacalao de profundidad en la subárea 48.3, durante la temporada 91/92.

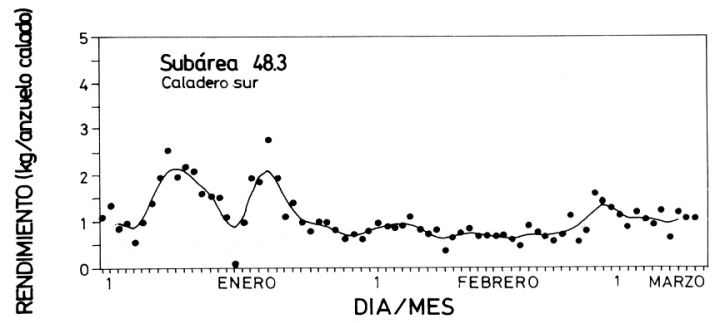

Figura 9. Rendimiento obtenido por la flota chilena en la captura de bacalao de profundidad en la subárea 48.3, durante la temporada 91/92. 


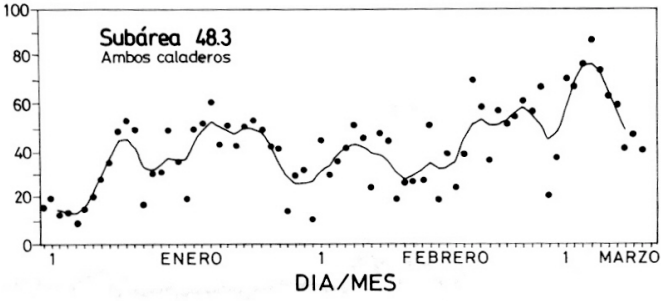

Figura 10. Captura de bacalao de profundidad registrada por la flota chilena en la subárea 48.3, durante la temporada 91/92.

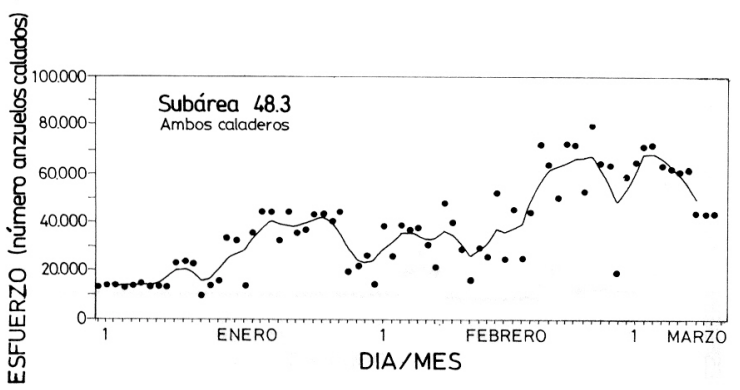

Figura 11. Esfuerzo aplicado por la flota chilena para la captura de bacalao de profundidad en la subárea 48.3, durante la temporada 91/92.

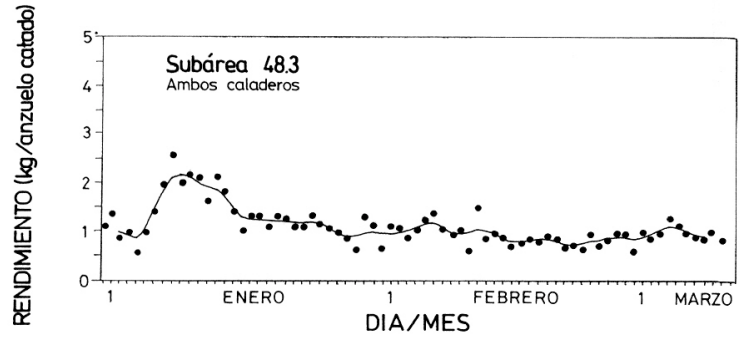

Figura 12. Rendimiento obtenido por la flota chilena en la captura de bacalao de profundidad en la subárea 48.3, durante la temporada 91/92.

\section{Evaluación del recurso y captura total permisible}

Los resultados obtenidos en el análisis de cohorte efectuado con las capturas extraídas en la temporada 1991/1992, indican que la biomasa total explotable está comprendida entre 19.861 y 33.804 ton, para tasas de mortalidad natural de 0,06 y 0,18 respectivamente. Valores intermedios, se obtienen al efectuar los cálculos con las mortalidades naturales consideradas como más probables $(0,10 ; 0,11$ y 0,12$)$, con las cuales la población estimada fluctúa entre 23.443 y 25.575 ton (Tabla 3 ).

De acuerdo a los efectivos por edad obtenidos por el método anterior y utilizando el mismo rango de mortalidades naturales anteriores, pero combinados con edades de primera captura entre 5 y 10 años o corregidas en 6 a 11 años, la CTP calculada para 1992/1993, fluctúa entre 2.630 y 4.957 ton en los machos, y de 2.752 a 7.353 ton en las hembras. Puntualmente, al considerar tc $=8$ o 9 años, una tasa de mortalidad natural de 0,11 y una mortalidad total intermedia al rango estimado a través de diferentes métodos (Tabla 1 ), $\mathrm{Z}=0,40$ en machos y $\mathrm{Z}=0,32$ en hembras, se obtuvo una captura total permisible calculada en forma global cercana a las 8.000 ton (Tabla 4).

Por otra parte, al aplicar el método de DeLury, los resultados proporcionaron una población explotable total entre 11.671 y 11.690 ton. Dichos valores, fueron obtenidos corrigiendo las capturas acumuladas en número de ejemplares con mortalidades naturales en el rango 0,06 y 0,18 y empleando un peso promedio por ejemplar de 11,3 kg. El valor medio se obtuvo al considerar $\mathrm{M}=0,11$, resultando una población de 11.680 ton (Fig. 16 y Tabla 5).

Finalmente, al proyectar la CTP para la temporada 1992/1993 a partir de la biomasa estimada por el método de DeLury y utilizando un valor de $\mathrm{F}_{0,1}$ estimado por el método de Beverton y Holt, se obtuvo una CTP que fluctuóentre 1.085 y 1.262 ton para $\mathrm{M}=0,11$ y tc=8-10 o 9-10 años (Tabla 6). Sin embargo, si se aplica el criterio $\mathrm{F}_{0,1}$ calculado por Thompson y Bell, utilizando los mismos valores en los parámetros, se obtienen capturas recomendables que varían entre 4.304 y 5.768 ton.

\section{DISCUSION}

Como una manera de conocer y orientar la toma de decisiones en esta pesquería, en la que participan 


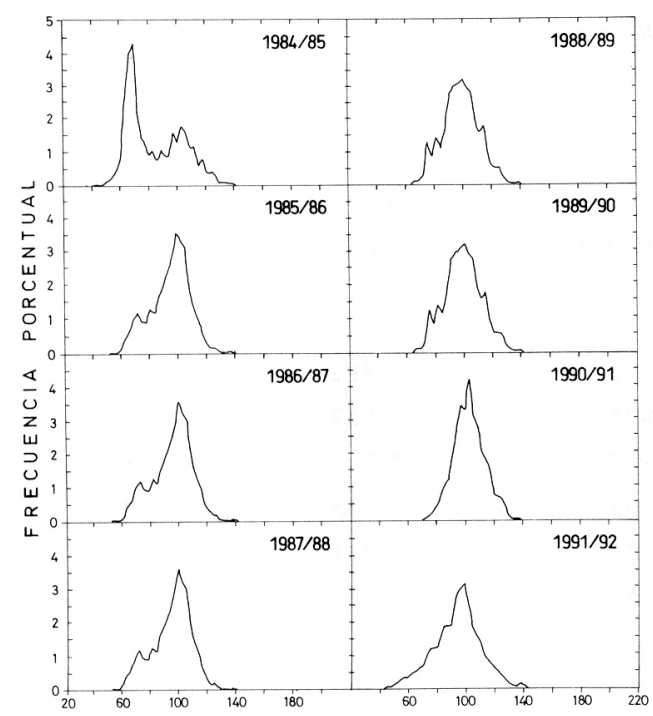

Figura 13. Machos: Distribuciones de frecuencias de tallas de las capturas de bacalao de profundidad, registradas en la subárea 48.3 desde la temporada 1984/ 85 hasta la temporada 1991/92. Fuente: Gasyukov (1991) y Arana et al. (1992)

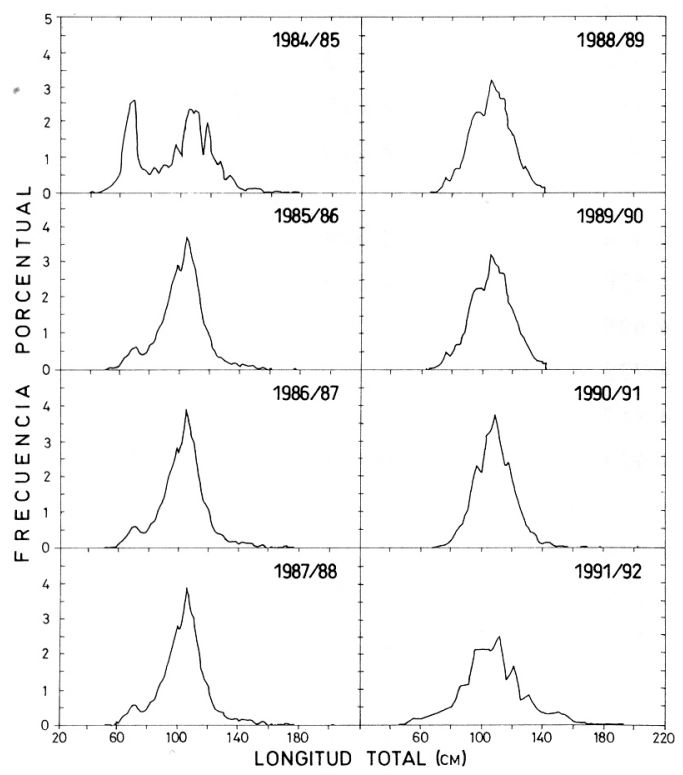

Figura 14. Hembras: Distribuciones de frecuencias de tallas de las capturas de bacalao de profundidad, registradas en la subárea $\mathbf{4 8 . 3}$ desde la temporada 1984/85 hasta la temporada 1991/92. Fuente: Gasyukov (1991) y Arana et al. (1992)

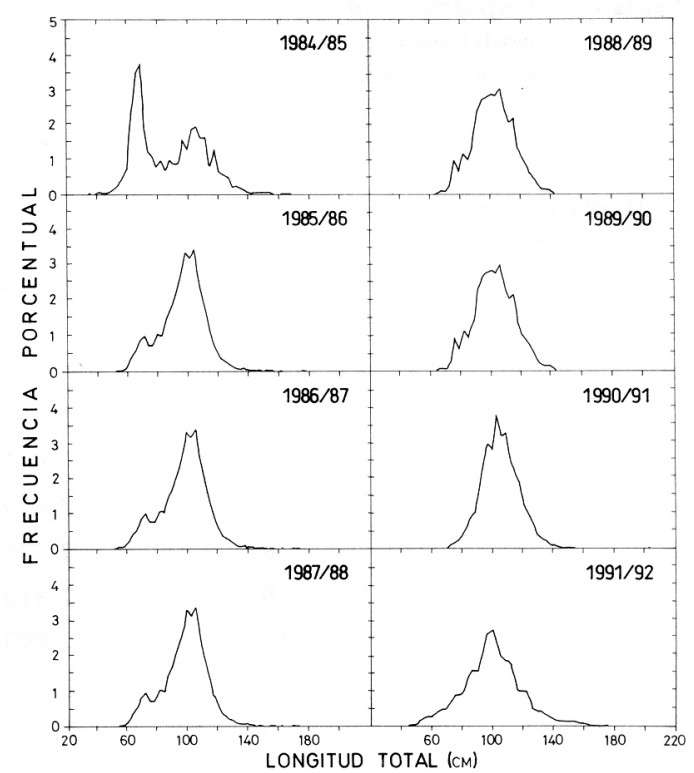

Figura 15. Ambos sexos: Distribuciones de frecuencias de tallas de las capturas de bacalao de profundidad, registradas en la subárea 48.3 desde la temporada 1984/ 85 hasta la temporada 1991/92. Fuente: Gasyukov (1991) y Arana et al. (1992)

Tabla 3. Biomasa explotable de machos y hembras de bacalao de profundidad, calculadas con diferentes mortalidades naturales a través del análisis de cohorte (temporada 1992/1993).

\begin{tabular}{cccc}
\multirow{2}{*}{$\begin{array}{c}\text { MORTALIDAD } \\
\text { NATURAL } \\
(\mathrm{M})\end{array}$} & $\begin{array}{c}\text { BIOMASA EXPLOTABLE } \\
\text { (ton) }\end{array}$ & $\begin{array}{c}\text { BIOMASA } \\
\text { GLOBAL } \\
\text { (ton) }\end{array}$ \\
\cline { 2 - 3 } & MACHO & HEMBRA & \\
\hline 0,06 & 09.670 & 10.191 & 19.861 \\
0,10 & 11.252 & 12.191 & 23.443 \\
0,11 & 11.701 & 12.776 & 24.477 \\
0,12 & 12.173 & 13.401 & 25.575 \\
0,18 & 15.602 & 18.202 & 33.804 \\
\hline
\end{tabular}


Tabla 4. Capturas totales permisibles de machos y hembras de bacalao de profundidad, calculadas con diferentes mortalidades naturales y edades de primera captura, basado en el análisis de cohorte utilizando $\mathrm{F}_{0,1}$ obtenido por el método de Thompson y Bell (Ricker, 1975)

\begin{tabular}{|c|c|c|c|c|c|}
\hline \multirow{2}{*}{$\begin{array}{l}\text { MORTALIDAD } \\
\text { NATURAL } \\
\text { (M) }\end{array}$} & \multirow{2}{*}{$\begin{array}{c}\text { EDAD PRIMERA } \\
\text { CAPTURA } \\
\text { CALCULADA } \\
\text { (años) }\end{array}$} & \multicolumn{2}{|c|}{ C T P (ton) } & \multirow{2}{*}{$\begin{array}{c}\text { C T P } \\
\text { GLOBAL } \\
(\text { ton })\end{array}$} & \multirow{2}{*}{$\begin{array}{c}\text { EDAD } \\
\text { PRIMERA } \\
\text { CAPTURA } \\
\text { CORREGIDA } \\
\text { (años) }\end{array}$} \\
\hline & & MACHO & HEMBRA & & \\
\hline \multirow{6}{*}{0.06} & 5 & 2.630 & 2.752 & 5.382 & 6 \\
\hline & 6 & 2.647 & 2.875 & 5.522 & 7 \\
\hline & 7 & 2.679 & 2.987 & 5.666 & 8 \\
\hline & 8 & 2.735 & 3.103 & 5.838 & 9 \\
\hline & 9 & 2.810 & 3.221 & 6.031 & 10 \\
\hline & 10 & 2.893 & 3.351 & 6.244 & 11 \\
\hline \multirow{6}{*}{0.10} & 5 & 3.192 & 3.755 & 6.947 & 6 \\
\hline & 6 & 3.209 & 3.875 & 7.084 & 7 \\
\hline & 7 & 3.240 & 3.989 & 7.229 & 8 \\
\hline & 8 & 3.294 & 4.096 & 7.390 & 9 \\
\hline & 9 & 3.364 & 4.203 & 7.567 & 10 \\
\hline & 10 & 3.444 & 4.320 & 7.764 & 11 \\
\hline \multirow[t]{6}{*}{0.11} & 5 & 3.354 & 4.969 & 7.423 & 6 \\
\hline & 6 & 3.371 & 4.186 & 7.557 & 7 \\
\hline & 7 & 3.401 & 4.295 & 7.696 & 8 \\
\hline & 8 & 3.454 & 4.378 & 7.832 & 9 \\
\hline & 9 & 3.522 & 4.498 & 8.020 & 10 \\
\hline & 10 & 3.600 & 4.610 & 8.210 & 11 \\
\hline \multirow{6}{*}{0.12} & 5 & 3.526 & 4.414 & 7.940 & 6 \\
\hline & 6 & 3.542 & 4.526 & 8.068 & 7 \\
\hline & 7 & 3.572 & 4.629 & 8.201 & 8 \\
\hline & 8 & 3.593 & 4.723 & 8.316 & 9 \\
\hline & 9 & 3.660 & 4.817 & 8.477 & 10 \\
\hline & 10 & 3.736 & 4.922 & 8.658 & 11 \\
\hline \multirow{6}{*}{0.18} & 5 & 4.804 & 7.248 & 12.052 & 6 \\
\hline & 6 & 4.817 & 7.261 & 12.078 & 7 \\
\hline & 7 & 4.838 & 7.275 & 12.113 & 8 \\
\hline & 8 & 4.870 & 7.291 & 12.161 & 9 \\
\hline & 9 & 4.909 & 7.313 & 12.222 & 10 \\
\hline & 10 & 4.957 & 7.353 & 12.310 & 11 \\
\hline
\end{tabular}


Tabla 5. Parámetros estimados en el ajuste regresional simple de DeLury, aplicado a las capturas de bacalao de profundidad (Dissostichus eleginoides) efectaudas en la isla Georgia del Sur durante la temporada 1991/1992

\begin{tabular}{cccccc}
\hline $\begin{array}{c}\text { MORTALIDAD } \\
\text { NATURAL }\end{array}$ & INTERSEPTO & PENDIENTE & $\begin{array}{c}\text { COEFICIENTE } \\
\text { CORRELACION }\end{array}$ & $\begin{array}{c}\text { TAMAÑO DE } \\
\text { LA MUESTRA }\end{array}$ & $\begin{array}{c}\text { BIOMASA } \\
\text { ESTIMADA (ton) }\end{array}$ \\
\hline$M=0,06$ & 117.568 & $-0,1136$ & 0,633 & 15 & 11.690 \\
$M=0,10$ & 117.568 & $-0,1137$ & 0,633 & 15 & 11.684 \\
$M=0,11$ & 117.568 & $-0,1137$ & 0,633 & 15 & 11.680 \\
$M=0,12$ & 117.568 & $-0,1137$ & 0,633 & 15 & 11.680 \\
$M=0,18$ & 117.568 & $-0,1138$ & 0,633 & 15 & 11.671 \\
\hline
\end{tabular}

Tabla 6. Capturas totales permisibles combinadas para ambos sexos de bacalao de profundidad, calculadas con diferentes mortalidades naturales y edades de primera captura, basándose en las biomasas obtenidas por el método de DeLury y utilizando $F_{0,1}$ calculados por Beverton y Holt (1975)

\begin{tabular}{|c|c|c|c|c|c|c|c|}
\hline \multirow{2}{*}{$\begin{array}{c}\text { EDAD DE } \\
\text { PRIMERA } \\
\text { CAPTURA } \\
\text { CALCULADA } \\
\text { (años) }\end{array}$} & \multirow{2}{*}{$\begin{array}{l}\text { LONGITUD } \\
\text { PRIMERA } \\
\text { CAPTURA } \\
(\mathrm{cm})\end{array}$} & \multicolumn{5}{|c|}{ MORTALIDAD NATURAL } & \multirow{2}{*}{$\begin{array}{c}\text { EDAD DE } \\
\text { PRIMERA } \\
\text { CAPTURA } \\
\text { CORREGIDA } \\
\text { (años) }\end{array}$} \\
\hline & & $M=0.06$ & $\mathrm{M}=0.10$ & $\mathrm{M}=0.11$ & $\mathrm{M}=0.12$ & $\mathrm{M}=0.18$ & \\
\hline 5 & 58.8 & 520 & 764 & 826 & 889 & 1.275 & 6 \\
\hline 6 & 68.5 & 557 & 838 & 909 & 981 & 1.438 & 7 \\
\hline 7 & 77.4 & 596 & 913 & 996 & 1.078 & 1.603 & 8 \\
\hline 8 & 85.4 & 637 & 992 & 1.085 & 1.178 & 1.767 & 9 \\
\hline 9 & 92.7 & 679 & 1.072 & 1.174 & 1.276 & 1.924 & 10 \\
\hline 10 & 99.4 & 720 & 1.151 & 1.262 & 1.374 & 2.072 & 11 \\
\hline
\end{tabular}

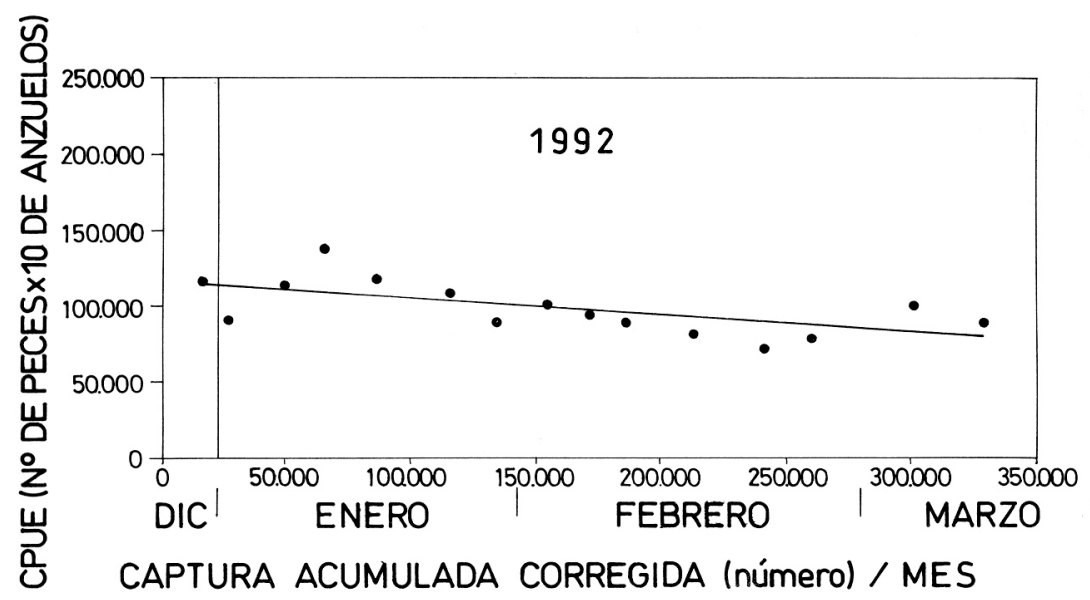

Figura 16. Cálculo de la biomasa del bacalao de profundidad (Dissostichus eleginoides) de la isla Georgia del Sur, a través del método regresional simple de DeLury relacionando las CPUE con las capturas acumuladas corregidas $(M=0,11)$, efectuadas durante la temporada 1991/1992. 
por primera vez embarcaciones chilenas, este estudio se orientó a describir el marco global en que se desenvuelve esta actividad y establecer los principales aspectos biológico-pesqueros relacionados con el bacalao de profundidad (Dissostichus eleginoides). No obstante, lo anterior, se debe considerar que los trabajos realizados se enmarcan en el procesamiento y análisis de la información disponible, recolectada de las faenas extractivas realizadas por la flota comercial que operó en la subárea 48.3 (isla Georgia del Sur), entre diciembre de 1991 y marzo de 1992.

Al comparar la CPUE, entre la presente temporada y las cifras de rendimientos entregadas pbr la CCRVMA comprendidas entre 1989, año en que comienza a utilizarse principalmente el espinel, y 1991, se desprende que en la presente temporada se registraron los valores más altos que se hayan producido históricamente en los rendimientos por anzuelo (Fig. 17). A modo descriptivo, se destaca que la CPUE promedio de enero a marzo de 1992 fue de 1,06 kg/ anzuelo, mientras que en igual período de 1989, 1990 y 1991, dichos valores, calculados en la misma unidad a partir de un peso promedio individual informado de $10,82 \mathrm{~kg}$; alcanzaron a sólo 0,$81 ; 0.74$ y 0.68 $\mathrm{kg} /$ anzuelo, respectivamente.

Al analizar los rendimientos estacionales obtenidos en la temporada de pesca 1991/1992, se aprecia que éstos fueron disminuyendo en forma gradual de enero a marzo, hecho que concuerda con la tenden- cia observada en los últimos años (CCRVMA, 1991). De esto se deduce que el incremento paulatino en las capturas durante este período sólo se debió a un aumento del esfuerzo aplicado y a una mayor eficiencia de la flota nacional.

Por otra parte, es importante destacar la imprecisión existente en los valores de diversos parámetros, como son principalmente los de crecimiento, talla de primera madurez sexual y longitud de primera captura. Esto, a su vez, hace que los cálculos en que se ocupan estos parámetros presenten un cierto grado de incertidumbre, lo que también conduce a notables variaciones entre autores que, al ocupar diferentes valores o combinación de ellos, obtienen resultados discrepantes. De allí es que en este documento se ha preferido presentar los resultados, utilizando rangos de valores de ingreso, a fin de establecer la sensibilidad de los resultados al cambiar los parámetros de entrada.

Al analizar la mortalidad por pesca óptima calculada por el método de Thompson y Bell, se observó que con una tasa de mortalidad natural y edades de primera captura consideradas más probables $(\mathrm{M}=0,11$ y tc=8-9 años o 9-10 años en edad corregida), el $\mathrm{F}_{0,1}$ de machos y hembras es superior al calculado en forma global por Gasyukov et al. (1991). Esta diferencia se hace más notoria si se considera que dichos autores trabajaron con $\mathrm{M}=0,18$. Lo contrario, sucede al estimar este parámetro con el método de

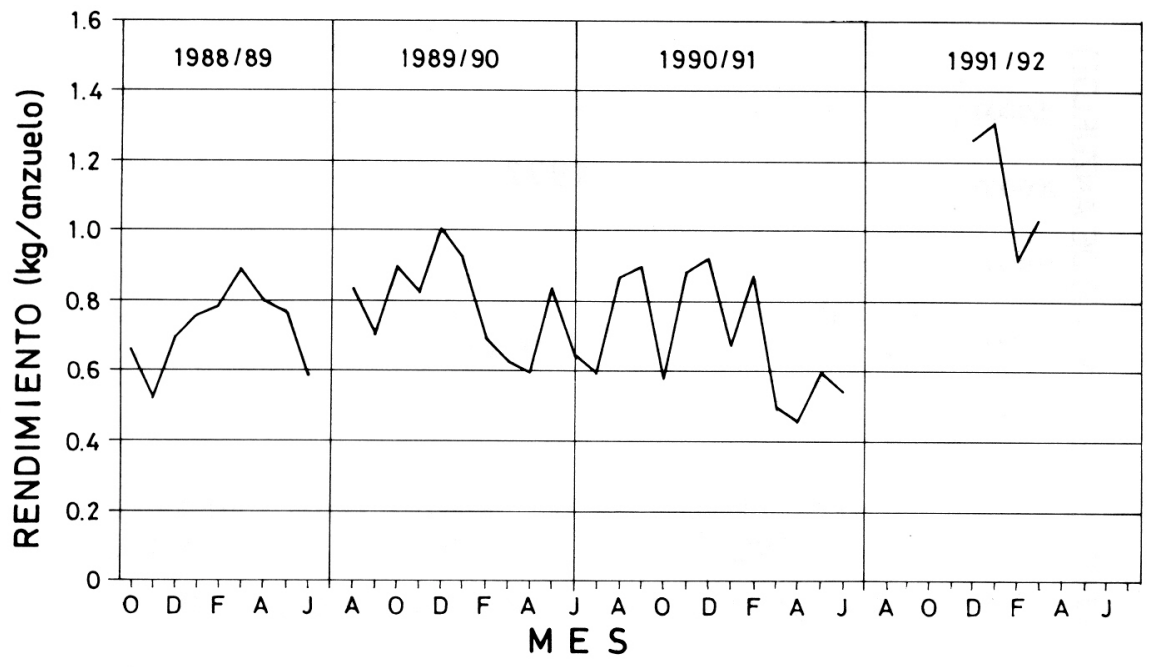

Figura 17. Captura por unidad de esfuerzo (kg/anzuelo) de bacalao de profundidad (Dissostichus eleginoides) obtenidas en la subárea 48.3 (isla Georgia del Sur), en diferentes temporadas de pesca. 
Beverton y Holt (1957), ya que la tasas calculadas de esta manera no sobrepasan los 0,22. Sin embargo, estos valores se aproximan bastante a los $\mathrm{F}_{0,1}$ utilizados por el Comité Científico (CCRVMA, 1991), al proponer las capturas para la temporada 1991/1992 basándose en la biomasa estimada por el método de DeLury.

En el ámbito de la evaluación de la población a través de este último procedimiento, es destacable el hecho de encontrar una buena correlación entre los rendimientos de pesca expresados en ejemplares por millón de anzuelos y las capturas acumuladas en número, corregidas por M. Asimismo, es importante mencionar que los coeficientes de capturabilidad (q) y de correlación (r) encontrados en este caso, son similares a los obtenidos con datos de la temporada 1990/1991 (CCRVMA, 1991). Sin embargo, estacoincidencia no se observó en la estimación de la biomasa proyectada para la temporada entrante (1992/1993), dado que en este estudio resultó superior en alrededor del $30 \%$ a la hallada en el período anterior. Sin embargo, cabe señalar que la población estimada por este método es levemente superior a la calculada por Everson (1991) a través de standing stock, aunque hay que considerar que el resultado obtenido en su trabajo es altamente incierto, como lo señala el mismo autor.

Si se considera que no existen evidencias para inferir que la población se encontraría en aumento, la discrepancia observada se debería en parte, a los mayores índices de rendimiento alcanzados por la flota chilena durante 1991/1992 y, por otro lado, al mayor peso promedio estimado en las capturas efectuadas por estos buques, equivalente a $11,3 \mathrm{~kg}$, a diferencia de $10 \mathrm{~s} 10,8 \mathrm{~kg}$ determinados en la temporada extractiva precedente.

A pesar del razonable ajuste obtenido y a la aparente congruencia observada al aplicar el método de DeLury, existe el inconveniente que se estaria subestimado el nivel poblacional, ya que dadas las características de las faenas de pesca con anzuelos, la captura por unidad de esfuerzo empleada (CPUE), no representaría efectivamente un índice de abundancia. Cabe destacar, que dicho coeficiente es alterado principalmente por la saturación que se produce en el anzuelo al retener un ejemplar, impidiendo la captura de otros individuos que se encuentren en el área de influencia de la carnada. Así también, el desprendimiento prematuro de la camada del anzuelo, constituiría otro factor que estaría alterando la obtención de un verdadero indicador de biomasa. Además, los rendimientos de captura se ven considerablemente afectados de acuerdo al tipo de anzuelo y carnada que se utilice (Arana et al., 1992).

De acuerdo al razonamiento anterior, la proyección de la CTP basándose en el método de DeLury, también estaría subevaluada, impidiendo determinar de manera óptima la verdadera capacidad de respuesta que el recurso tendría ante la presión de pesca. En ese sentido, al adoptar una cuota de captura fundamentada en este acercamiento metodológico, la medida de manejo tendría una posición destacadamente conservadora.

Por otra parte, al efectuar la evaluación a través del análisis de cohortes, se encontró la limitante de no disponer de información que permitiera construir una matriz captura-edad que incluyera varias temporadas de pesca. Sin embargo, se consideró que la información generada por la flota chilena durante 1991/ 1992, es lo suficientemente precisa y detallada como para ser utilizada en un análisis de cohortes que considerase únicamente el vector de captura-edad generado en ese período. Al proceder de esta manera, los resultados obtenidos en el análisis de cohorte, mostraron una biomasa explotable inferior a la señalada en trabajos anteriores (Shust et al., 1990 y Gasyukov et al., 1991). En dichos estudios, en que se utilizó una mortalidad natural de 0,18 , se obtuvo una cantidad de biomasa que dobla la calculada en el presente trabajo.

En parte, esta discrepancia se debería a la no incorporación en los cálculos de ejemplares menores de 5 años y mayores de 18 años de edad, ya que no estuvieron presentes en las capturas efectuadas en la temporada pasada. Otra causa que provocaría esta diferencia, estaría relacionada con una variación del patrón de explotación, vale decir que se habrían modificado las mortalidades por pesca en los diferentes grupos de edades, motivado fundamentalmente por las naves espineleras chilenas, que operaron por primera vez en la zona. Cabe señalar, que estas embarcaciones obtuvieron en forma notoria mayores rendimientos por unidad de esfuerzo, respecto a los observados históricamente en esa misma subárea (Fig. 17).

A pesar de la menor biomasa explotable calculada para 1991/1993, la captura total permisible basada en el análisis de cohorte alcanza una cifra similar a las calculadas para las temporadas 1989/1990 y 1991/ 1992 por Shust et al. (1990) y Gasyukov et al. (1991), determinándose por tercera vez consecutiva una CTP 
Tabla 7. Estimados de biomasa y capturas totales permisibles (CTP) para el bacalao de profundidad en el área 48.3 (Isla Georgia del Sur). (Modificado de CCRVMA, 1992).

\begin{tabular}{|c|c|c|c|c|c|}
\hline \multirow{2}{*}{ METODO } & \multirow{2}{*}{ PARAMETRO } & \multirow{2}{*}{$\begin{array}{l}\text { BIOMASA } \\
\text { (ton) }\end{array}$} & \multicolumn{3}{|c|}{ C T P (ton) } \\
\hline & & & $\begin{array}{l}\mathrm{F}_{\mathrm{O}, 1}=0,104 \\
(\mathrm{M}=0,10)\end{array}$ & $\begin{array}{l}F_{O, 1}=0,119 \\
(M=0,13)\end{array}$ & $\begin{array}{l}\mathrm{F}_{\mathrm{O}, 1}=0,136 \\
(\mathrm{M}=0,16)\end{array}$ \\
\hline DeLury (toda la temporada) & - & 1.200 & 1.130 & 1.260 & 1.430 \\
\hline DeLury (densidad local) & - & 9.800 & 920 & 1.030 & 1.170 \\
\hline \multicolumn{6}{|l|}{ Ancho teórico lateral de } \\
\hline \multirow[t]{4}{*}{ "influencia" del espinel } & $1 \mathrm{mn}$ & 8.000 & 750 & 840 & 950 \\
\hline & $0,50 \mathrm{mn}$ & 16.000 & 1.500 & 1.690 & 1.910 \\
\hline & $0,25 \mathrm{mn}$ & 32.000 & 3.000 & 3.360 & 3.800 \\
\hline & $0,25 \mathrm{mn}$ & 160.000 & 15.000 & 16.900 & 19.090 \\
\hline Radio teórico de & $10 \mathrm{~m}$ & 102.000 & 9.600 & 10.070 & 2.170 \\
\hline \multirow[t]{3}{*}{ "influencia" del anzuelo } & $15 \mathrm{~m}$ & 45.000 & 4.230 & 4.740 & 5.370 \\
\hline & $20 \mathrm{~m}$ & 25.000 & 2.350 & 2.630 & 2.980 \\
\hline & $25 \mathrm{~m}$ & 19.000 & 1.790 & 2.000 & 2.270 \\
\hline \multirow{3}{*}{$\begin{array}{l}\text { Análisis de cohorte basado } \\
\text { en la longitud }\end{array}$} & $M=0,10$ & 36.000 & 3.387 & - & - \\
\hline & $M=0,13$ & 61.000 & - & 6.426 & - \\
\hline & $M=0,16$ & 119.000 & - & - & 14.201 \\
\hline Análisis de cohorte basado & $\mathrm{M}=0,10$ & 23.443 & 2.009 & - & - \\
\hline en la edad (Arana et al., & $\mathrm{M}=0,13$ & 26.741 & - & 2.485 & - \\
\hline 1992) & $M=0,16$ & 30.707 & - & - & 3.080 \\
\hline
\end{tabular}

cercana a las 8.000 ton (Tabla 4). Dicha compensación, se sustentaría en gran medida en la mayor tasa de mortalidad por pesca óptima obtenida en este trabajo, en comparación a los $\mathrm{F}_{0,1}$ calculados para las temporadas anteriores.

Recientemente, el Grupo de Evaluación de Stock de Peces, reunido en Robart (Tasmania, Australia, 13-22 octubre 1992), definió una mortalidad natural de $\mathrm{M}=0,13$ como más probable para este recurso y efectuó estimaciones de la biomasa disponible de D. eleginoides en los alrededores de la isla Georgia del Sur a través de diversos métodos (Tabla 7). Entre ellos se encuentran los métodos de DeLury y análisis de cohorte basados en la edad y en las longitudes, ya discutidos anteriormente. Conjuntamente, se utilizaron otras dos metodologías aproximativas, en atención a la incertidumbre existente en la información biológica-pesquera de esta especie y en la no del todo bien conocida selectividad de los espineles.

De este modo, se intentó obtener índices de densidad en este recurso para proyectar la biomasa de acuerdo al área total de distribución de estos peces. Con este fin se efectuaron dos aproximaciones metodológicas: la primera de ellas, de acuerdo al área de pesca o de influencia del espinel como un todo (área rectangular), y la segunda considerando la posible área de influencia de cada anzuelo (área circular). En el primer caso se estima una influencia lateral del espinel, proyectando una cierta distancia a cada lado de la línea madre y, en el segundo, se considera un radio tentativo de atracción o de posibilidad de encuentro entre los peces y el anzuelo.

En el caso del área de operación del espinel como un todo, las consideraciones son únicamente teóricas, ya que no existen elementos técnicos para establecer la distancia lateral de atracción o encuentro 
entre los peces y la componente cebo-anzuelo. De allí es que se definieran arbitrariamente dichas distancias para sensibilizar densidades aproximadas, utilizando las capturas efectuadas durante la temporada 1991/92.

En la opción de establecer densidad de bacalao de profundidad por unidad de área mediante la segunda aproximación, se utilizaron los registros de CPUE disponible de la flota chilena. Para ello se consideró el promedio de 1,06 kg/anzueloy el peso medio de $11,3 \mathrm{~kg}$ por ejemplar capturado, lo cual indica un éxito de captura de un pez por cada 10,7 anzuelos, resultado semejante al obtenido por Duhamel (1992) en faenas de pesca experimental realizadas en la isla Kerguelén. De acuerdo a este índice y a la distancia entre reinales, se puede estimar el radio teórico de captura de un bacalao, lo que podria ser interpretado como la presencia de un ejemplar en una determinada superficie circular. Al utilizar este valor es posible obtener un índice de abundancia que puede ser proyectado al área total de distribución de la especie para estimar, de este modo, la biomasa disponible.

Con los resultados obtenidos, el Grupo de Evaluación de Stock de Pesca consideró que «una biomasa superior a las 45.000 toneladas no sería realista». Por ello, estableció un rango de Cuota Total Permisible similar a la establecida en la temporada 1991/92, vale decir entre 750 y 5.370 ton. Así, basada en los antecedentes disponibles, la CCRVMA fijó una cuota de 3.350 ton para ser capturada en la subárea administrativa 48.3 entre el 6 de diciembre de 1992 y la próxima reunión de esta Comisión.

Finalmente, el grupo técnico de trabajo sugirió que no se incremente el esfuerzo aplicado en esta pesquería, a fin de evitar el introducir complicaciones en las estimaciones de CPUE y con ello en las evaluaciones de stock que se realicen posteriormente. De este modo, se podría evitar que la pesquería se cierre prontamente. De allí la recomendación de la CCRVMA de restringir el ingreso de nuevos buques a esta pesquería, motivo por el cual deberian operar durante la temporada 1992/93 alrededor de la isla Georgia del Sur y Shag Rocks (Subárea 48.3) únicamente las naves que cuentan con autorizaciones vigentes.

\section{REFERENCIAS BIBLIOGRAFICAS}

AGUAYO, M. y L. CID. 1991. Recopilación, proceso y análisis de los antecedentes biológico-pesquero en la pesca exploratoria de bacalao de profundidad realizada por el B/P Friosur V. Inst. Fom. Pesq., Informe interno, $63 \mathrm{pp}$.

ARANA, P; M. ARREDONDO y V. VENTURINI. 1992. Análisis biológico-pesquero de las capturas de bacalao de profundidad (Dissostichus eleginoides), efectuadas en el sector Atlántico del océano Austral durante la temporada 1991/1992. Estud. Doc., Univ. Católica Valparaíso, 21/92: 90 pp.

ARREDONDO, M. 1991. Utilización de registros de producción de buques espineleros congeladores en estudios biológico-pesqueros de la merluza austral (Merluccius australis). Tesis, Esc. Ciencias del Mar, Univ. Católica Valparaíso, 92 pp.

BEVERTON, R. y S. HOLT. 1956. A review of methods for estimating mortality rates in fish population, with special reference to source of bias in catch sampling. Rapp. P-V. Reun. Cons. Penn. Int. Explor. Mer, 140: 67-83.

BEVERTON, R. y S. HOLT. 1957. On the dynarnics of the exploited fish populations. U.K. Min. Agr. Fish. and Food, Fish. Invest., Ser. II, 533 pp.

Convención para la Conservación de los Recursos Vivos Marinos Antárticos (CCRVMA). 1990. Informe del grupo de trabajo para la evaluación de poblaciones de peces. SC-CAMLR-IX: 1-150.

Convención para la Conservación de los Recursos Vivos Marinos Antárticos (CCRVMA). 1991. Informe del grupo de trabajo para la evaluación de poblaciones de peces. SC-CAMLR-X: 217-363.

Convención para la Conservación de los Recursos Vivos Marinos Antárticos (CCRVMA). 1992. Informe del grupo de trabajo para la evaluación de poblaciones de peces. SC-CAMLR-XI: 93-361.

CHAPMAN, D.G. 1972. The whale problem: a status reporto Schevill, W.E. (Ed.).

DUHAMEL, G. 1992. Exploratory longline fishing around the Kerquelen islands (Division 58.5.1). Description of the fishing effort; catchability and target size of Dissostichus eleginoides. CCAMLR, WG-FSA-92/31 (Rev. 1): 1-11. 
EVERSON, I. 1991. Stock assesment of the Patagonian toothfish (Dissostichus eleginoides), at South Georgia. CCAMLR, WG-FSA-91/20: 1-21.

GAETE, V. y P. ARANA. 1985. Análisis de la proporción sexual en la langosta de Juan Femández (Jasus frontalis). In: «Investigaciones Marinas en el Archipiélago de Juan Fernández», P. Arana (Ed.), Escuela de Ciencias del Mar, UCV, Valparaíso, pp. 213-223.

GARCIA, S. y L. LE RESTE. 1987. Ciclos vitales, dinámica, explotación y ordenación de las poblaciones de camarones penéidos costeros. FAO Doc. Tec. Pesca, 203: 1-180.

GASYUKOV, PS.; R.S. DOROVSKIKH and K.V. SHUST. 1991. Assessment of the Dissostichus eleginoides stock in area 48.3 for the 1990/1991 season and calculation of TAC for the 1991/1992 season. CCAMLR, WG-FSA-91/24: 1-31.

HUREAU, J.C. and C. OZOUF-COSTAZ. 1980/81. Age determination and growth of Dissostichus eleginoides Smitt, 1898 from Kerguelen and Crozet Islands. Cybium, 3 sér., 3(8): 23-32.

JONES, R and N. VAN ZALINGE. 1982. Estimates of mortality rate population size for shrimp in Kuwait waters. Kuwait Bull. Mar. Sci., 2: 273-288.

PAULY, D. 1980. On the interrelationships between natural mortality, growth parameters, and mean environmental temperature in 175 fish stocks. J. Cons. Int. Explor. Mer, 39(2): 175-192.

PAULY, D. 1983. Length-converted catch curves: a power-full tool for fisheries research in the tropics (Part I). Fishbyte, 2(1): 9-13.

Recibido el 15 de diciembre de 1993. Aceptado el 2 de noviembre de 1994.
PAULY, D. 1984. Length-converted catch curves: a power-full tool for fisheries research in the tropics (Part II). Fishbyte, 2(1): 17-19.

POPE, J.G. 1972. An investigation of the accuracy of virtual population analysis using cohort analysis. Res. Bull. ICNAF, (9): 65-74.

RICKER, W. 1975. Computation and interpretation of biological statistics of fish population. Bull. Fish. Res. Bd. Can., 191: 382 pp.

RIKHTER, V. and V. EFANOV. 1976. On one aproaches to estimation of natural mortality of fish populations. ICNAF Res. Doc., 76/VI/8: $12 \mathrm{pp}$.

ROFF, D. 1984. The evolution of life history parameters in teleosts. Can. J. Fish. Aquat. Sci., 41: 969-1000.

SHUST, K.V; PS. GASYUKOV; RS. DOROVSKIKH and B.A. KENZHIN. 1990. The state of Dissostichus eleginoides stock and TAC for 1990/91 in subarea 48.3 (South Georgia). CCAMLR, WG-FSA-90/34: 1-16.

SSENTONGO, G. and P. LARKIN. 1973. Some simple methods of estimating mortality rates of exploited fish populations. J. Fish. Res. Bd. Can., 30(5): 695698.

THOMPSON, W.F. and F.H. BELL. 1934. Biological statistics of the Pacific halibut fishery. 2. Effect of changes in intensity upon total yield and yield per unit of gear. Rep. Int. Fish. (Pacific Halibut) Comm., (8): 49 pp.

VAN SICKLE, J. 1977. Mortality rates fron size distributions. The aplication of a conservation law. Oecología (Berlin), 27: 311-318. 